\title{
Acute lymphoblastic leukemia in a 2-year-old girl whose mother was previously diagnosed with antiphospholipid syndrome: a case report
}

\author{
Miguel Ángel Castro-Jiménez ${ }^{1,2^{*}}$, Carlos Efraín Cortés-Sánchez ${ }^{3}$, Ernesto Rueda-Arenas ${ }^{4}$ \\ and Lucy Adela Tibaduiza-Buitrago ${ }^{1}$
}

\begin{abstract}
Background: The role of maternal exposures and conditions in the origin of childhood cancer has been a subject of growing interest, but current evidence is inconclusive.

Case presentation: We present a case detected in a multicenter case-control study evaluating the association between parental risk factors and childhood acute lymphoblastic leukemia (ALL). The patient is a Colombian girl who was diagnosed with ALL-L1 when she was 2 years old. Her mother had been diagnosed with antiphospholipid syndrome before pregnancy and had also been treated with subcutaneous injections of heparin. Other potentially relevant maternal and patient exposures are also reported in this paper.
\end{abstract}

Conclusion: We hypothesize that the maternal autoimmune disease could be a contributor in the causality network of the daughter's leukemia. However, the role of other exposures cannot be excluded.

Keywords: Acute lymphoblastic leukemia, Child, Case report, Antiphospholipid syndrome, Maternal exposure

\section{Background}

This case was detected in a multicenter case-control study evaluating the association between parental risk factors and childhood acute lymphoblastic leukemia (ALL) $[1,2]$. In Western countries, ALL is the most common malignant disease in children under the age of 15 [3]. The etiologic factors contributing to the development of ALL have not been completely established, but include exposure to benzene, ionizing radiation, and some genetic syndromes [4]. Over the last few decades, interest has grown in the effect that certain parental exposures before birth or conception might have on the risk of childhood ALL [5-10]. Several environmental exposures and clinical conditions have been studied as hypotheses, but the results of these studies have been inconsistent [11-20]. Additionally, some authors have

\footnotetext{
* Correspondence: mcastro2505@yahoo.es

'Grupo Colombiano de Estudios Alfa en Epidemiología, Salud Poblacional, Estadística Aplicada y Ciencias Aliadas. Magna Science Corporation, Bogotá, D.C., Colombia

${ }^{2}$ Grupo de Investigación Epidemiológica del Cáncer, Instituto Nacional de Cancerología, Avenida Calle 1 No. 9-85, Bogotá, D.C., Colombia

Full list of author information is available at the end of the article
}

recently explored genetic variants and their role in the early stages of this type of leukemia [21-23]. Addressing the association between uncommon maternal disorders, such as some immunological diseases, and ALL in the offspring, which also has a low incidence, presents difficulties because of the low number of possible comparisons. We present a case of ALL in a girl whose mother had been previously diagnosed with a chronic immunological disease and discuss other potentially associated maternal and child exposures during three time periods: the last 24 months prior to the estimated date of conception of the patient, prenatal growth, and childhood development.

\section{Case presentation}

The patient is a Colombian girl who was diagnosed at the age of 2 with the precursor $\mathrm{B}$ (common) form of ALL-L1 (CD10+, CD13+) with a normal karyotype. No molecular analysis was performed. The patient's mother had been diagnosed with antiphospholipid syndrome (APS) and treated with subcutaneous injections of heparin (of unknown periodicity) since she was 26 . The mother 
was 30 years old at the child's birth and her blood type was A positive. She had undergone three spontaneous abortions before giving birth to her only live child (the case patient) and had been diagnosed with venous thrombosis when she was 16 .

In the last 24 months before conception, the child's mother was exposed to diagnostic X-rays in her abdominal region three times and took medications such as acetaminophen and nonsteroid anti-inflammatory drugs (NSAIDs). During the pregnancy there were various high-risk conditions such as threatened abortion, fetal distress, gestational diabetes, premature rupture of the membranes, and preterm labor. The mother took the prescribed prenatal multivitamin, supplemental ferrous pills, and acetaminophen daily. She also took a NSAID 1 day per month during the first 4 months of pregnancy and received a subcutaneous injection of heparin twice daily throughout the pregnancy.

The mother was not actively exposed to cigarette smoke, alcohol, or psychoactive drugs during the preconception or prenatal periods. However, she was exposed to passive smoking 7 days a week (less than five cigarettes per day) before the pregnancy.

The patient was born by cesarean section. Her weight, height, and blood type were $2240 \mathrm{~g}, 47 \mathrm{~cm}$, and $\mathrm{AB}$ positive, respectively. At birth she was diagnosed with neonatal jaundice and unspecified problems with breathing. She was breastfed for 18 months. Prior to being diagnosed with ALL, the case patient presented with septic arthritis and was hospitalized. During this period she received several types of antibiotics (unspecified) and possibly other medications. She had been exposed to diagnostic $\mathrm{X}$-rays seven times before the diagnosis of ALL for assessment of her thorax $(n=3)$, abdomen $(n=2)$, and right leg $(\mathrm{n}=3)$. The patient was a passive smoker from birth until her diagnosis of ALL $(<5$ cigarettes per day/7 days per week). During all study periods (i.e., preconception, index pregnancy, and postnatal life), the case patient and/or her mother were continuously exposed to chemical products including thinner, gasoline, paints, and solvents because there was a woodworking shop in the building where they lived. At the time of the mother's interview the patient was alive.

\section{Conclusions}

Some parental exposures have been associated with an increased risk of childhood ALL $[1,7,14]$. In this report we have presented a case that is possibly related to a chronic maternal condition with alteration of immunological functions, its long-term treatment, or both. Previous studies have shown an association between childhood cancer and autoimmune disease in the patient [24] and between childhood cancer and a history of immunological disease such as APS in the parent [25-27]. Both APS and childhood ALL are diseases with low incidence rates in the general population. In fact, APS is estimated to have an incidence of approximately five new cases and a prevalence of 40 cases per 100,000 persons [28]. Therefore, studying the effects of uncommon maternal diseases on the risk of childhood ALL in children who were "exposed" to them, even prior to conception, represents a problem with respect to etiological evaluation because of the lack of an adequate sample size in terms of statistical power to confirm or discard any association.

We hypothesized that maternal APS might be related to the onset of ALL in this patient, although the causative role of other maternal and child exposures such as medications, passive smoking, and hydrocarbons (and other chemical products) cannot be excluded. Regarding the exposure to several X-rays, we need to take into account that this procedure was performed for the radiologic diagnosis or follow-up of recurrent infections that could be the first complications of an undiagnosed leukemia. Thus, it is possible that at least some of the $\mathrm{X}$-rays were ordered after the actual onset of the leukemia, reducing the probability of a causal role in this case. Further epidemiologic studies designed to assess the relationship between maternal disorders such as APS and childhood ALL are needed.

\section{Consent}

Written informed consent was obtained from the patient's parents for publication of this Case Report and any accompanying images. A copy of the written consent is available for review by the Editor-in-Chief of this journal. This Case Report is based on a patient of a Colombian case-control study previously approved by the Industrial University of Santander Ethical Committee, the Javeriana University Ethical Committee, and the Institutional Review Boards of the participating hospitals.

\section{Abbreviations}

ALL: Acute lymphoblastic leukemia; NSAID: Nonsteroid anti-inflammatory drug; APS: Antiphospholipid syndrome.

\section{Competing interests}

The authors declare that they have no competing interests.

\section{Authors' contributions}

MACJ designed the original study and proposed an early version of this paper. CECS assessed the exposure to some chemical products. ERA was a clinical investigator of the original study and LATB contributed with a clinical and epidemiological view of the case. All of the authors made substantial contributions to the analysis and interpretation of data for this report, reviewed it critically, and approved the final version. All authors read and approved the final manuscript.

\section{Acknowledgements}

This paper is a product of a study that was partly supported by grants from COLCIENCIAS (no. 106-2003), the National Cancer Institute of Colombia (no. 0402-2004, Terry Fox Run), Universidad Industrial de Santander (Bucaramanga, Colombia), and Magna Science Corporation (Bogotá, Colombia) 


\section{Author details}

'Grupo Colombiano de Estudios Alfa en Epidemiología, Salud Poblacional, Estadística Aplicada y Ciencias Aliadas. Magna Science Corporation, Bogotá, D.C., Colombia. ${ }^{2}$ Grupo de Investigación Epidemiológica del Cáncer, Instituto Nacional de Cancerología, Avenida Calle 1 No. 9-85, Bogotá, D.C., Colombia. ${ }^{3}$ Postgrado en Salud Ocupacional. Universidad El Bosque, Avenida Carrera 9 No. 131 A - 02, Edificio Fundadores, Bogotá, D.C., Colombia. ${ }^{4}$ Departamento de Pediatría, Facultad de Salud. Universidad Industrial de Santander - Hospital Universitario de Santander, Carrera 33 No. 20-126., Bucaramanga, (Santander), Colombia.

Received: 7 March 2014 Accepted: 31 March 2015

Published online: 15 April 2015

\section{References}

1. Castro-Jiménez MA, Orozco-Vargas LC. Parental exposure to carcinogens and risk for childhood acute lymphoblastic leukemia, Colombia, 2000-2005. Prev Chronic Dis. 2011;8(5):A106. http://www.cdc.gov/pcd/issues/2011/sep/ 10_0201.htm. Accessed 25 Aug 2013.

2. Castro-Jiménez MA. Exposiciones preconcepcionales y prenatales ¿están asociadas con leucemia linfoblástica aguda en los menores de 15 años? Bucaramanga: Universidad Industrial de Santander; 2007. http://repositorio. uis.edu.co/jspui/bitstream/123456789/10006/2/122658.pdf. Accessed 20 Jul 2013].

3. Ferlay J, Shin HR, Bray F, Forman D, Mathers C, Parkin DM. GLOBOCAN 2008 V2.0, Cancer Incidence and Mortality Worldwide: IARC CancerBase No. 10 [Internet]. Lyon, France: International Agency for Research on Cancer; 2010 http://globocan.iarc.fr. Accessed 13 Jul 2013.

4. Petridou E, Trichopoulos D. Leukemias. In: Adami HO, Hunter D, Trichopoulos D, editors. Textbook of Cancer Epidemiology. Oxford: Oxford University Press; 2002. p. 556-8.

5. Fabia J, Thuy TD. Occupation of father at time of birth of children dying of malignant diseases. Br J Prev Soc Med. 1974;28(2):98-100.

6. Infante-Rivard C, Siemiatycki J, Lakhani R, Nadon L. Maternal Exposure to Occupational Solvents and Childhood Leukemia. Environ Health Perspect. 2005;113:787-92.

7. McKinney PA, Fear NT, Stockton D, On behalf of the UK childhood cancer study investigators. Parental occupation at periconception: findings from the United Kingdom Childhood Cancer Study. Occup Environ Med. 2003;60:901-9.

8. Jensen CD, Block G, Buffler P, Ma X, Selvin S, Month S. Maternal dietary risk factors in childhood acute lymphoblastic leukemia (United States). Cancer Causes Control. 2004;15:559-70.

9. Milne E, Greenop KR, Scott RJ, Bailey HD, Attia J, Dalla-Pozza L, et al. Parenta prenatal smoking and risk of childhood acute lymphoblastic leukemia. Am J Epidemiol. 2012;175(1):43-53.

10. Glass DC, Reid A, Bailey HD, Milne E, Fritschi L. Risk of childhood acute lymphoblastic leukaemia following parental occupational exposure to pesticides. Occup Environ Med. 2012;69(11):846-9.

11. Hasle $\mathrm{H}$, Clemmensen $\mathrm{IH}$, Mikkelsen M. Risk of leukaemia and solid tumors in individuals with Down's syndrome. Lancet. 2000;355:165-9.

12. Koushik A, King WD, McLaughlin JR. An ecologic study of childhood leukemia and population mixing in Ontario, Canada. Cancer Causes Control. 2001;12:483-90.

13. Greaves MF. Childhood Leukemia. BMJ. 2002;324:283-7.

14. Gardner MJ, Snee MP, Hall AJ, Powell CA, Downes S, Terrell JD. Results of case-control study of leukaemia and lymphoma among young people near Sellafield nuclear plant in West Cumbria. BMJ. 1990;300:423-9.

15. Roman E, Doyle P, Maconochie N, Davies G, Smith PG, Beral V. Cancer in children of nuclear industry employees: report on children aged under 25 years from nuclear industry family study. BMJ. 1999;318:1443-50.

16. Greaves M. Infection, immune responses and the aetiology of childhood leukaemia. Nature Rev Cancer. 2006;6:193-203.

17. Kinlen $L$. Epidemiological evidence for an infective basis in childhood leukaemia. Br J Cancer. 1995;71:1-5.

18. Kinlen LJ. Childhood leukemia and population mixing. Pediatrics. 2004;114:330-1.

19. Chan LC, Lam TH, Li CK, Lau YL, Li CK, Yuen HL, et al. Is the timing of exposure to infection a major determinant of acute lymphoblastic leukemia in Hong Kong? Paediatr Perinat Epidem. 2002;16:154-65.
20. Petridou E, Dalamaga M, Mentis A, Skalkidou A, Moustaki M, Karphatios T, et al. Evidence on the infectious etiology of childhood leukemia: the role of low herd immunity. Cancer Causes Control. 2001;12:645-52.

21. Migliorini G, Fiege B, Hosking FJ, Ma Y, Kumar R, Sherborne AL, et al. Variation at 10p12.2 and 10p14 influences risk of childhood B-cell acute lymphoblastic leukemia and phenotype. Blood. 2013;122(19):3298-307.

22. Treviño LR, Yangw, French D, Hunger $S$, Carroll WL, Devidas M, et al. Germline genomic variations associated with childhood acute lymphoblastic leukemia. Nat Genet. 2009;41(9):1001-5.

23. Papaemmanuil E, Hosking FJ, Vijayakrishnan J, Price A, Olver B, Sheridan E, et al. Loci on 7p12.2, 10q21.2 and $14 q 11.2$ are associated with risk of childhood acute lymphoblastic leukemia. Nat Genet. 2009;41(9):1006-10.

24. van de Schans SA, van Spronsen DJ, Hooijkaas H, Janssen-Heijnen ML, Coebergh JW. Excess of autoimmune and chronic inflammatory disorders in patients with lymphoma compared with all cancer patients: a cancer registry-based analysis in the south of the Netherlands. Autoimmun Rev. 2011;10(4):228-34.

25. Mellemkjaer L, Alexander F, Olsen JH. Cancer among children of parents with autoimmune diseases. Br J Cancer. 2000;82(7):1353-7.

26. Till M, Rapson N, Smith PG. Family studies in acute leukaemia in childhood: a possible association with autoimmune disease. Br J Cancer. 1979;40(1):62-71.

27. Wen $W Q$, Shu XO, Sellers T, Bhatia S, Lampkin B, Robison LL. Family history of cancer and autoimmune disease and risk of leukemia in infancy: a report from the Children's Cancer Group (United States and Canada). Cancer Causes Control. 1998;9(2):161-71.

28. Gómez-Puerta JA, Cervera R. Diagnosis and classification of the antiphospholipid syndrome. J Autoimmun. 2014;48-49:20-5.

\section{Submit your next manuscript to BioMed Central and take full advantage of:}

- Convenient online submission

- Thorough peer review

- No space constraints or color figure charges

- Immediate publication on acceptance

- Inclusion in PubMed, CAS, Scopus and Google Scholar

- Research which is freely available for redistribution

Submit your manuscript at www.biomedcentral.com/submit 